\title{
Dilemma Hartz IV: Geringverdiener entlasten
}

Als der damalige Bundeskanzler Gerhard Schröder im September 2002 die Bundestagswahl gewann, hatte die Hartz-Kommission gerade ihre Ergebnisse vorgelegt. Schröder versprach, die Vorschläge 1:1 umzusetzen und kündigte Einschnitte im Sozialbereich an. Das Hartz-Konzept wurde in vier Stufen umgesetzt (Hartz I bis IV) und trat 2003 bis 2005 in Kraft. Insbesondere Hartz IV wurde wegen der Zusammenlegung von Sozialhilfe und Arbeitslosenhilfe zum Arbeitslosengeld II und aufgrund der damit verbundenen Absenkung von Leistungen kritisiert. Heute ist die Debatte um die Leistungen nach dem SGB II (Hartz IV) zurückgekehrt. Die Leistungen seien zu niedrig, auf Sanktionen solle verzichtet, und Hinzuverdienstmöglichkeiten verbessert werden. Befürworter der Hartz-Reformen argumentieren, dass niedrige Leistungen und der damit verbundene Lohnabstand (Abstand zwischen Höhe des Nettolohns und Transferleistung) Anreize zur Aufnahme einer Tätigkeit gewährleisten. Die Sanktionen wiederum dienen dazu, den Lohnabstand im Bedarfsfall zu vergrößern. Befürworter weisen auch auf den deutlichen Rückgang der Arbeitslosenquote seit Einführung der Leistungen hin. Diese sank von 2005 bis 2018 von $11,7 \%$ auf 5,2 \%; in Ostdeutschland sogar von $18,7 \%$ auf 6,9\%. Kritiker wiederum ordnen die Ursache für diesen Rückgang nicht den Reformen zu. Vielmehr sei der deutsche Arbeitsmarkt von einem weltweiten Aufschwung erfasst worden.

Zum historischen Kontext gehört auch, dass Deutschland 2003 als „kranker Mann Europas" galt, von 2001 bis 2005 das Defizitkriterium des Maastricht-Vertrags nicht einhalten konnte, und nach Wegen suchte, das Defizit wieder unter die $3 \%$-Grenze zu führen. Hinzu kam eine Debatte über mangelnde Wettbewerbsfähigkeit. Die Löhne seien zu hoch und eine Verbesserung der Wettbewerbsfähigkeit in der Währungsunion allein über eine interne Abwertung möglich. Zur Ausgaben- und Lohnsenkung wären Leistungskürzungen ein probates Mittel. Dieser Kontext hat sich seither allerdings verändert. So wird die Arbeitslosigkeit in Deutschland heute nicht mehr als das zentrale Problem gesehen. Die öffentlichen Haushalte weisen seit Jahren Überschüsse aus. 2018 betrugen sie insgesamt $1,7 \%$ des Bruttoinlandsprodukts und fielen damit rund 6 Prozentpunkte höher aus als 2003. Gleichzeitig haben sich die Überschüsse in der Leistungsbilanz ebenfalls um 6 Prozentpunkte erhöht, was verstärkt kritisiert wird. Ein Wettbewerbsfähigkeitsproblem wird heute nicht mehr gesehen. Diskussionen um Verteilungsgerechtigkeit und Niedriglohnbeschäftigung nehmen heute einen größeren Raum ein als zu Beginn des Jahrtausends. Hinzu kommen Anreizkonflikte, die mit dem Arbeitslosengeld II verbunden sind und als „Armutsfallen“ beschrieben werden. Sie betreffen insbesondere die mangelnden Hinzuverdienstmöglichkeiten: Empfänger von Leistungen des SGB II können durch Hinzuverdienst kaum ihr Haushaltseinkommen steigern, da ein erheblicher Teil des Hinzuverdienstes mit den Leistungen verrechnet wird. Zudem wirken auch andere Anrechnungstatbestände anreizfeindlich. So reduziert die Prüfung von Vermögensgrenzen Anreize zur privaten Vorsorge und auch die Anrechnung der Einkommen von Haushaltsmitgliedern führt zu Fehlanreizen.

Zudem bereiten die Empfänger-Zahlen Sorgen: Während die Arbeitslosigkeit von 2005 bis 2018 deutlich zurückgeführt wurde, stagnierte die Zahl der SGB-II-Empfänger bei rund 6 Mio. Betroffenen. Das Arbeitslosengeld II ist schon deshalb fragwürdig, weil es nicht mehr nur Arbeitslosengeld ist. Lediglich 1,7 Mio. Bezieher von ALG II sind tatsächlich arbeitslos. Der größere Teil der erwerbsfähigen Leistungsberechtigten ist in Erwerbstätigkeit (1,2 Mio.), in arbeitsmarktpolitischen Maßnahmen (0,6 Mio.), in Schule, Studium oder Ausbildung (0,4 Mio.) und anderen Sondersituationen. Die rund 6 Mio. Betroffenen bestehen vor allem aus ca. 2 Mio. Kindern bzw. 2,6 Mio. Personen unter 25

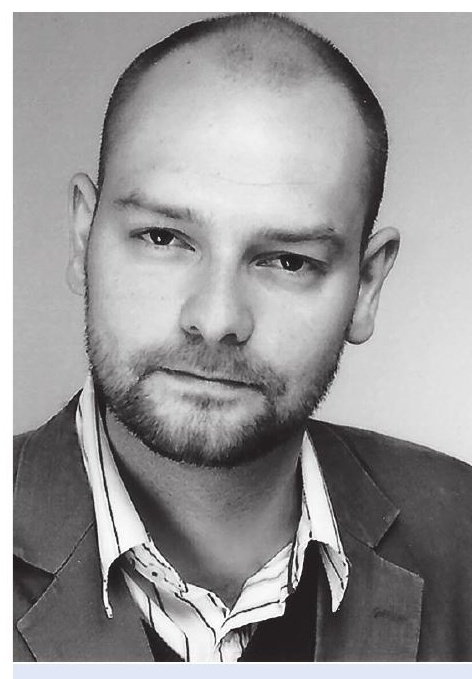

Christian Breuer ist Juniorprofessor für Europäische Wirtschaft an der Technischen Universität Chemnitz. 
Jahren, sowie 0,6 Mio. Alleinerziehenden. Dabei verändert sich die Gruppe der erwerbsfähigen Leistungsberechtigten seit 2005. Die Zahl der Personen über 55 Jahre nimmt zu (von 0,6 auf 0,8 Mio.) und ebenso die Zahl der erwerbsfähigen Ausländer (von 0,9 Mio. auf 1,5 Mio.). Kritisch ist, dass die Vermittlung in den Arbeitsmarkt häufig nicht mit einer existenzsichernden Beschäftigung einhergeht, die die Betroffenen in die Lage versetzt, ohne Lohnzuschüsse auszukommen, und sich so Armutsfallen verfestigen.

Insofern ist es nachvollziehbar, dass Politiker verschiedener Parteien derzeit an Vorschlägen zur Reform von Hartz IV arbeiten. Allerdings sollten dabei auch ökonomische Aspekte bedacht werden. So können Leistungserhöhungen Armutsfallen vergrößern. Zudem würde sich die Zahl der SGB-II-Bezieher erhöhen, was angesichts der Anreizkonflikte nicht wünschenswert erscheint. Andere Vorschläge sehen die Abschaffung von Sanktionen und Anrechnungen (etwa bei Vermögen) vor. Dies würde zwar einige Anreizkonflikte mildern, allerdings entspräche eine völlig anrechnungs- und sanktionsfreie Auszahlung der Grundsicherung de facto einem bedingungslosen Grundeinkommen. Dabei dürften derzeit die rein monetären Anreize zur Arbeitsaufnahme nicht ausreichen, da die Betroffenen gegenwärtig nur $10 \%$ bis $20 \%$ vom Hinzuverdienst behalten dürfen. Ausweichreaktionen in die Schattenwirtschaft wären zu erwarten.

Aktuelle Vorschläge sehen daher vor, den Transferentzug bei Hinzuverdienst auf $70 \%$ statt bisher $80 \%$ bis $90 \%$ zu reduzieren, was den Kreis der Anspruchsberechtigten deutlich vergrößern dürfte. Studien zeigen bei $70 \%$ Transferentzug, dass Singles mit einem Bruttoeinkommen in Höhe von 2000 Euro und Alleinerziehende bis 4000 Euro in die Grundsicherung fielen. Dies wiederum kann nur funktionieren, wenn alle weiteren Anrechnungstatbestände und Sanktionen entfielen, denn wie wäre es zu begründen, dass sich neben den 6 Mio. Betroffenen weitere Millionen Geringverdiener mit der Bürokratie des SGB II befassen sollten. Statt aber Niedriglöhne bei Hinzuverdienst aufzustocken, könnte der gleiche Effekt auch erreicht werden, wenn die Einkommen der Geringverdiener spürbar entlastet würden. Die Grenzbelastung jedes hinzuverdienten Euro ab 450 Euro beträgt heute bereits $30 \%$ bis $40 \%$ allein beim Arbeitnehmer, da die Einkommen oberhalb der Minijobs einer sehr hohen Belastung durch Sozialbeiträge unterliegen. Hinzu kommt die Belastung durch Steuern ab etwa 800 Euro. Unnötig erscheint, solche Einkommen mit üppigen Transferleistungen aufzustocken und diese gleichzeitig zu rund $50 \%$ mit Abgaben zu belasten. Der einzige Effekt wäre eine Erhöhung der Staatsquote.

Viel einfacher wäre, die Grundsicherung und die Belastung mit Steuern und Beiträgen zu verrechnen und die Nettoeinkommen durch Entlastungen außerhalb des Transfersystems zu stärken. Ein Freibetrag für Sozialbeiträge in Höhe von 450 oder 850 Euro könnte die Mini- und Midijobs ersetzen und den Lohnabstand herbeiführen, an dem es im bisherigen System mangelt. Dies würde das Nettoeinkommen um etwa 100 bis 200 Euro pro Monat und Beschäftigten erhöhen und Anreize zur Beschäftigung außerhalb des SGB II setzen. Ein Halbtagsjob würde für Singles bei gegebenem Mindestlohn ausreichen, um über dem Hartz-IV-Niveau zu liegen. Für Familien oder Alleinerziehende sind die Leistungen nach dem SGB II derzeit sehr hoch. Hier entstehen Armutsfallen durch den Entzug der Regelleistungen für Kinder bei Hinzuverdienst, da das Kindergeld deutlich niedriger ausfällt als die Leistungen des SGB II, sowie über höhere Kosten der Unterkunft. Ein höheres Kindergeld könnte die Familieneinkommen außerhalb des SGB-II-Bezugs stärken und den Kreis der Empfänger von Arbeitslosengeld II deutlich reduzieren, z.B. im Fall von Alleinerziehenden. Wünschenswert wäre eine Diskussion darüber, wie neben der Arbeitslosigkeit auch die Zahl der Empfänger von Leistungen des SGB II reduziert werden könnte. Dabei sollte darüber nachgedacht werden, wie die Nettoeinkommen von Geringverdienern und Familien außerhalb der Grundsicherung erhöht werden könnten, um Armutsfallen aufzulösen. Am besten erfolgt eine solche Dis-

Technische Universität Chemnitz christian.breuer@wirtschaft.tu-

chemnitz.de kussion in einer entsprechenden Reformkommission. 